\title{
Assessing the Role of Social Media in the Fight Against Sexual Assault
}

\section{Chinyere Linda Agbasiere ${ }^{1}$}

\author{
${ }^{1}$ Madonna University \\ P. M. B 05 Elele, Rivers State, Nigeria
}

DOI: $10.22178 /$ pos.76-7

JEL Classification: I39

Received 21.10.2021

Accepted 25.11.2021

Published online 30.11 .2021

Corresponding Author: agbasierechinyere@gmail.com

(C) 2021 The Author. This article is licensed under a Creative Commons Attribution 4.0 License @ (1)
Abstract. We examine the challenges faced by women for effective communication on social media. The majority of the data used in this study was primary, and it was gathered in the field using a questionnaire. The questionnaire utilized in this study was a written list of questions that had been carefully designed and selected to collect data. The questions were closed-ended and focused on women's issues and their use of social media. Cronbach alpha was used to test the dataset's validity, consistency, and reliability based on the obtained information. Cronbach's Alpha for all constructs or items was more significant than the minimum acceptable reliability coefficient of 0.5 to 0.9 , indicating strong internal consistency.

Furthermore, Cronbach's Alpha for all constructs was more than the minimal permissible reliability coefficient, indicating that they were valid and trustworthy and hence suitable for use. We notice that most respondents in this study reported that they had experienced social media harassment. Our findings show, in particular, that the majority of respondents in this study believe that women are usually overlooked when it comes to matters requiring social media opinions. We opined that information, education, and communication are empowering tools from the preceding. It is the challenge of women in leadership to dismantle the obstacles in the women's use of information media. No form of women empowerment will succeed until these challenges are met.

Keywords: social media; women empowerment; communication; gender equity.

\section{INTRODUCTION}

Violence against women (VAW) is one of the most systematic and prevalent human rights abuses globally. Such gender-based violence against women is a form of discrimination deeply rooted in power imbalances and structural inequality between women and men. Violence against women is a global phenomenon, occurring in every continent, country, and culture. It harms families, impoverishes communities, and reinforces other forms of inequality and violence throughout societies.

Sexual assault continues to be a pervasive problem in the United States. While prevalence estimates vary considerably based on research samples and definitions of sexual assault, a nationally representative survey estimates that $19.3 \%$ of women and $1.7 \%$ of men have been sexually assaulted at some point in their lifetime [1]. A significant body of research has been devoted to examining the typical characteristics of male perpetrators who sexually assault female victims. A deeper understanding of the characteristics most frequently associated with sexual assault perpetrators helps understand the etiology of sexual assault perpetration. In addition, it may help identify possible methods of intervention and prevention.

Several non-governmental organizations (NGOs), international organizations, and government interventions have used mass media in the past few years to combat sexual harassment and other forms of VAW and promote family planning which is another essential sexual health issue through development communication [14]. However, most of these campaigns, especially recent ones, lack published data measuring their impacts.

Data on sexual violence typically come from the police, clinical settings, non-governmental organizations, and survey research. The relationship between these sources and the global magnitude of the problem of sexual violence may be viewed as corresponding to an iceberg floating in the water. Research on female sexual assault perpetra- 
tors is still emerging, with fewer studies than on male perpetrators who assault female victims. Most existing research has focused on the characteristics and behaviours of female offenders who commit assaults against children or adolescents. There is little consensus on the role of social media in combating sexual assaults, hence the motivation for this study.

The output of this study is a theoretical proposition aiming to identify ways in which online and social media could be used to combat sexual harassment on different levels, namely: the sexual harassment survivor; bystanders/witnesses of sexual harassment, or the immediate social circles of survivors and perpetrators; the Nigerian society at large; different organizations such as educational institutions, workplaces, malls, etc.; and the country's legislation and policies. Together, these propositions form a framework that other organizations and entities could utilize in Nigeria for development communication campaigns against sexual harassment. Ultimately, the use of this framework shall play a role in creating sexual harassment-free spaces and decreasing sexual harassment prevalence rates in Nigeria.

\section{Literature review}

Social media has gained a lot of popularity over the past few years. As a result of this popularity, other traditional Media have experienced a decline in business and popularity. Social media networks are applications that allow users to build personal websites accessible to other users for the exchange of unique content and communication [9]. According to [9], social media can be characterized as online applications, platforms, and media to facilitate interactions, collaborations, and content sharing. Author [3] argues that though communication is the core dimension of social media networks, not all platform categories are equally suitable for all marketing objectives because most platforms are not equally well suited for information, collaboration, and cultivating relationships. Social networks are primarily for communication and exchanging ideas of interest among peer groups or communities. According to [5], however, a long-term friendship can be developed and maintained through frequent communication initiated by the marketer on the interactive social networks.

Furthermore, the vocal members become the opinion leaders. In this way, a collaboration be- tween the marketer and the online consumer/or prospect is developed. This means that there would be no severe engagement amongst the online communities without information flow within the communities and the brand.

Sexual harassment is defined as: "any form of unwanted words and/or actions of a sexual nature that violate a person's body, privacy, or feelings and make that person feel uncomfortable, threatened, insecure, scared, disrespected, startled, insulted, intimidated, abused, offended, or objectified". Sexual harassment is a global problem that women suffer from in developing developed countries. For example, studies have shown that $79 \%$ of women living in urban settings in India, $86 \%$ in Thailand, and $89 \%$ in Brazil have faced harassment or violence in public spaces [16]. Furthermore, a survey conducted in Afghanistan - a country where women are required to cover completely - found that $93 \%$ of the women were subjected to harassment in public spaces, $87 \%$ in workplaces, and $89 \%$ in educational institutions. The case in the United Kingdom is no better; $75 \%$ of women living in UK cities have been subjected to sexual violence or harassment in public areas, and $51 \%$ of female Londoners feel at risk of facing harassment in public transportation [16]. Table 1 below shows some prevalence rates of different forms of sexual harassment in several countries.

Recently, as online and social media became more accessible, popular, and ubiquitous, they have become a communication medium. This includes health issues, such as changing beliefs and providing tips about prevention of certain diseases [6], providing health tips [17], awareness about specific conditions and their risks, and understanding of health risks. It also includes means of empowering women such as [2, 8], as well as combating violence against women in different forms, such as female genital mutilation/cutting (FGM/C), sexual harassment, and domestic violence [11].

Theoretical Framework. Earlier models used in development interventions, such as the selfefficacy model, focused mainly on the individual. However, changing violent behaviour does not depend entirely on the individual but also on social and physical environments and the individual's interaction with them. As such, campaigns aiming to combat violent behaviour must address the different levels of influence that may lead to such behaviour, thus increasing the prob- 
ability of changing it. Therefore, the theoretical framework for this study is primarily based on the Social Ecological Model, which was first developed following World War I to understand the interrelations between different levels of influence on human behaviour. By the 1970s, Urie Bronfenbrenner had developed a conceptual, social-ecological model for human development and continued to revise it throughout his scholarly career until 2005. The concept behind Bronfenbrenner's model is that to understand human development, the whole environment where this development occurs and the person's interaction with it should be studied. He defines development as: "a lasting change in how a person perceives and deals with his environment".

Empirical Review. Several studies have found that individuals with access to different mediums (e.g. radio, television, print media, and the Internet) use other mediums to fulfil different needs. The Internet - is a medium for different types of communication; one-to-one communication (e. g. email, private messages), group communication (e.g. group chats, social media posts), and mass communication (online newspapers, IPTV) makes identifying uses of the Internet as a medium for communication more problematic than traditional mediums which usually support one type of communication [4]. According to [4], people use online media with standard communication tools to fulfil different needs in their daily lives. For example, web surfing is used in ways similar to newspapers and television for collecting information and entertainment. The Internet was found to be the medium used most for ordering information. Interpersonal platforms are used for social bonding, maintaining relationships, problem-solving, and persuasion. This shows that as people get used to specific media types, the way they use them evolves, as the diffusion of innovations concept suggests.

In the past decade, social media has become increasingly popular. Today, it is used to "pass the time, maintain relationships, meet new people, keep up with current trends, and gather social information" [13]. Although, in addition, social media has been linked to collecting political information and civic engagement, it has also been found that college students who use Facebook for politics and civic engagement are more likely to practice them offline [10]. In addition, it has been found that social media users usually do not use one form of social media but rather utilize different forms of online and offline communication.
Furthermore, new social media platforms are linked to social trends to maintain a specific "communication repertoire" [12]. One increasingly widespread use of online media is helpseeking, especially in the case of health issues. Research has suggested that four in every ten adults have used the Internet at least once in the previous year to seek health information. In addition, using health websites has had a positive effect on attitude and behaviour change.

Using such websites is linked to reducing anxiety and increasing self-efficacy [17]. With the emergence of social media platforms, the Internet has become a more important source for seeking help and collecting health-related information. For example, in their study, [7] have used Facebook, Twitter and Google search engine for a pilot campaign about maternity health and found that these platforms - especially Facebook and Google - have a broad reach and are a cheap means of communicating public health information. This was of particular importance since $45 \%$ of the media time for Americans was spent on the Internet and mobile devices [7], and $73 \%$ of the adults use social media [13]. However, it is essential to note that the Internet and social media are also abused to harass women. In fact, with the rise in the use of social media, sexual harassment has become more prevalent. There are different ways of doing so, including sending unwanted messages with sexual content, abusing someone's data and photographs, online stalking, and even stalking in person [11].

\section{METHODOLOGY}

Data for this paper are drawn from 50 respondents of a cross-section of service providers, public educators, activists, advocates, writers, and researchers engaged in various activities relating to social media and sexual assaults. The majority of these respondents were engaged in a survey on social media. Thus, participants were not random but found through nonprobability sampling. Such sampling is appropriate, given that this study seeks to gain a deeper understanding of the perspectives and work of those already committed to sexual assaults prevention, rather than generalizing to the population at large. However, the data used for this study were majorly primary, and these were obtained from the field through a questionnaire. The questionnaire used in this study took the form of a written list of questions, which were constructed and ade- 
quately selected to obtain information. The questions were closed-ended based on social media and sexual assaults. Cronbach alpha was then used to test for the dataset's validity, consistency, and reliability from the data obtained. All the constructs or items had Cronbach's Alpha above the minimum acceptable reliability coefficient between 0.5 to 0.9 and exhibited good internal consistency. Since all the constructs had Cronbach's Alpha above the minimum proper reliability coefficient, it can be considered valid and reliable, therefore suitable for usage.

\section{RESULTS AND DISCUSSION}

This section analyses the questionnaire administered and retrieved from the respondents, from which our recommendation and conclusion are drawn. A total of 50 questionnaires were sent out to the respondents for data generation, as shown in the table below.

Table 1 - Analysis of Questionnaire

\begin{tabular}{|l|l|l|}
\hline Questionnaires & Copies & Percentage \\
\hline Retrieved \& validated & 40 & 80 \\
\hline Un-retrieved & 2 & 4 \\
\hline Unvalidated & 8 & 16 \\
\hline Sent copies & 50 & 100 \\
\hline
\end{tabular}

From the table above, out of the 50 questionnaires that were sent, 40 of them were retrieved and validated. This represented $80 \%$ of the total questionnaire sent, and this was the number used for analysis in the subsequent sections that will follow. However, the table also shows that 2 of the questionnaires could not be retrieved while eight copies representing $16 \%$ could not be validated as some were incomplete.

Table 2 - Gender Distribution of Respondents

\begin{tabular}{|l|c|c|}
\hline \multicolumn{1}{|c|}{ Gender } & No & Percentage \\
\hline Males & 18 & 44 \\
\hline Females & 22 & 56 \\
\hline Total & 40 & 100 \\
\hline
\end{tabular}

Also, from the retrieved questionnaires and as seen from the table above, it is observed that 18 of the respondents were males, which represented $44 \%$ of the total questionnaire retrieved. Similarly, 22 of them described $56 \%$ of the whole questionnaire recovered.

The table 3 shows the respondents' responses related to the research question: what is the role of social media in combating sexual assaults?

Table 3 - Responses to Research Question 2

\begin{tabular}{|l|l|c|c|c|c|c|c|c|c|}
\hline \multirow{2}{*}{ No } & Questions & \multicolumn{5}{|c|}{ Responses } & \multicolumn{3}{|c|}{ Total } \\
\cline { 4 - 10 } & Yes & $\%$ & Undecided & $\%$ & No & $\%$ & Response & $\%$ \\
\hline 1 & $\begin{array}{l}\text { Through social media, I can confidently express my } \\
\text { opinion on social harassment issues }\end{array}$ & 37 & 93 & 0 & 0 & 3 & 7 & 40 & 100 \\
\hline 2 & $\begin{array}{l}\text { Social media is a veritable tool to draw the attention } \\
\text { of policy makers on sexual harassment }\end{array}$ & 36 & 92 & 0 & 0 & 4 & 8 & 40 & 100 \\
\hline 3 & $\begin{array}{l}\text { I have participated in various campaigns on social } \\
\text { media on sexual harassment and assaults }\end{array}$ & 38 & 95 & 0 & 0 & 2 & 5 & 40 & 100 \\
\hline
\end{tabular}

From the table, we observed that, on average, 37 respondents agreed that through social media, they could confidently express their opinion on social harassment issues. This represented about 93\% of the total respondents. This implies that most of the respondents in this study agreed that social media could positively influence the fight against sexual harassment. Similarly, about 3 of the respondents disagreed that they can confidently express their opinion on social harassment issues through social media. This represented about $7 \%$ of the entire respondents. Also, from the table, we observed that, on average, 36 respondents agreed that social media is a veritable tool to draw the attention of policymakers on sexual harassment. This represented about 92\% of the total respondents. This implies that the majority of the respondents in this study agreed that social media is a veritable tool to draw the attention of policymakers on sexual harassment.

Similarly, we find that about 4 of the respondents disagreed that social media is a veritable tool to draw the attention of policymakers on sexual harassment. This represented about $8 \%$ of the 
entire respondents. We also find that 38 respondents participated in various campaigns on social media on sexual harassment and assaults. This represented about $95 \%$ of the total respondents. This implies that most of the respondents in this study participated in various campaigns on social media on sexual harassment and assaults. Similarly, we find that about 2 of the respondents did not participate in various campaigns on social media on sexual harassment and assaults, representing $5 \%$ of the respondents.

Social mobilization aims to raise awareness on a particular issue to achieve social change/development. Social mobilization can be any action taken by groups with the same development objective to mobilize society to reach this objective. This action could be in the form of signing a petition, protesting, or creating a social media campaign. Based on the findings of this study, we find that social media is a veritable tool to draw the attention of policymakers on sexual harassment. Mainly, our results show that majority of the respondents in this study participated in various campaigns on social media on sexual harassment and assaults. Media advocacy campaigns are designed to address the "power gap" rather than the "information gap". Development of communication campaigns that address the information gap target individuals by raising awareness and providing knowledge about a specific behaviour without considering the sociopolitical conditions that lead to or encourage such behaviour.

On the other hand, media advocacy campaigns consider that negative behaviour is not necessarily a result of lack of knowledge, and therefore addresses the power gap by targeting society as a whole and focusing on the sociopolitical root causes that lead to such behaviour. It aims to change the environment that enables such behaviour and pressure decision-makers to change policies or enforce them. Media advocacy campaigns target external factors that might lead to this behaviour, such as "basic housing, employment, education, healthcare, and personal security" [15].

\section{CONCLUSIONS}

Sexual harassment has several negative physical and psychological impacts on women and economic effects on the country. It has a significant adverse effect on women's freedom of movement, pursuing education, careers and different potential opportunities, women's health and well-being, and interaction and presence in the social and public spheres. The data used for this study were majorly primary, and these were obtained from the field through a questionnaire. The questionnaire used in this study took the form of a written list of questions, which were constructed and adequately selected to obtain information. Cronbach alpha was then used to test for the dataset's validity, consistency, and reliability from the data obtained. All the constructs or items had Cronbach's Alpha above the minimum acceptable reliability coefficient between 0.5 to 0.9 and exhibited good internal consistency. We conclude that social media is a veritable tool to draw the attention of policymakers on sexual harassment. Mainly, our results show that majority of the respondents in this study participated in various campaigns on social media on sexual harassment and assaults. Furthermore, there is evidence that social media content plays a vital role in combating sexual harassment through social media by stirring discussions and mobilizing the community to further advocate against sexual harassment through hashtags. As such, this could be an exciting topic for future research.

\section{REFERENCES}

1. Breiding, M. J. (2015). Prevalence and characteristics of sexual violence, stalking, and intimate partner violence victimization-National Intimate Partner and Sexual Violence Survey, United States, 2011. American Journal of Public Health, 105(4), e11-e12. doi: 10.2105/ajph.2015.302634

2. Dalal, N. (2006). Finding people in images and videos (Doctoral dissertation), Institut National Polytechnique de Grenoble-INPG). Retrieved from https://tel.archives-ouvertes.fr/tel00390303/document 
3. Fauser, M. (2017). Mixed Methods and Multisited Migration Research: Innovations From a Transnational Perspective. Journal of Mixed Methods Research, 12(4), 394-412. doi: $10.1177 / 1558689817702752$

4. Flanagin, A., \& Metzger, M. (2006). Internet use in the contemporary media environment. Human Communication Research, 27(1), 153-181. doi: 10.1111/j.1468-2958.2001.tb00779.x

5. Gummesson, E. (2002). Relationship marketing and a new economy: it's time for de-programming. Journal of Services Marketing, 16(7), 585-589. doi: 10.1108/08876040210447315

6. Hay, J., Coups, E. J., Ford, J., \& DiBonaventura, M. (2009). Exposure to mass media health information, skin cancer beliefs, and sun protection behaviors in a United States probability sample. Journal of the American Academy of Dermatology, 61(5), 783-792. doi: 10.1016/j.jaad.2009.04.023

7. Huesch, M. D., Galstyan, A., Ong, M. K., \& Doctor, J. N. (2016). Using Social Media, Online Social Networks, and Internet Search as Platforms for Public Health Interventions: A Pilot Study. Health Services Research, 51, 1273-1290. doi: 10.1111/1475-6773.12496

8. Laizu, Z., Armarego, J., \& Sudweeks, F. (2010, December). Cognitive change in women's empowerment in rural Bangladesh. In 2010 13th International Conference on Computer and Information Technology (pp. 277-282). IEEE.

9. Palmer, A., \& Koenig-Lewis, N. (2009). An experiential, social network-based approach to direct marketing. Direct Marketing: An International Journal, 3(3), 162-176. doi: 10.1108/17505930910985116

10. Park, N., Kee, K. F., \& Valenzuela, S. (2009). Being Immersed in Social Networking Environment: Facebook Groups, Uses and Gratifications, and Social Outcomes. CyberPsychology \& Behavior, 12(6), 729-733. doi: 10.1089/cpb.2009.0003

11. Pavan, E. (2017). Internet intermediaries and online gender-based violence. London: Routledge:

12. Quan-Haase, A., \& Young, A. L. (2010). Uses and Gratifications of Social Media: A Comparison of Facebook and Instant Messaging. Bulletin of Science, Technology \& Society, 30(5), 350-361. doi: $10.1177 / 0270467610380009$

13. Quinn, N. (Ed.). (2016). Finding culture in talk: A collection of methods. N. d.: Springer, 2016.

14. Rizzo, H., Price, A., \& Meyer, K. (2012). Anti-sexual harrassment campaign in Egypt. Mobilization: An International Quarterly, 17(4), 457-475.

15. Wallack, L. (1994). Media Advocacy: A Strategy for Empowering People and Communities. Journal of Public Health Policy, 15(4), 420. doi: 10.2307/3343024

16. Wilkinson, A. L., El-Hayek, C., Fairley, C. K., Roth, N., Tee, B. K., McBryde, E., ... Stoové, M. (2017). Measuring Transitions in Sexual Risk Among Men Who Have Sex With Men: The Novel Use of Latent Class and Latent Transition Analysis in HIV Sentinel Surveillance. American Journal of Epidemiology, 185(8), 627-635. doi: 10.1093/aje/kww239

17. Ybarra, M. L., \& Suman, M. (2006). Help seeking behavior and the Internet: A national survey. International Journal of Medical Informatics, 75(1), 29-41. doi: 10.1016/j.ijmedinf.2005.07.029 